Revista Arbitrada Interdisciplinaria de Ciencias de la Salud. SALUD Y VIDA

Volumen 3. Número 6. Año 3. Julio - Diciembre 2019

Hecho el depósito de Ley: FA2016000010

ISSN: 2610-8038

FUNDACIÓN KOINONIA (F.K)

Santa Ana de Coro, Venezuela.

Edisson Andrés Mogollón Guzmán; Manuel Alejandro Brito Vizuete; Miguel Mateo Sarmiento Álvarez; Johanna Paulina Estrada Cherres

DOI: http://dx.doi.org/10.35381/s.v.v3i6.344

\title{
Síndrome de Arteria Mesentérica Superior o Síndrome de Wilkie Caso Clínico
}

\section{Superior Mesenteric Artery Syndrome or Wilkie Syndrome Clinical case}

\author{
Edisson Andrés Mogollón Guzmán \\ mogollon2002@gmail.com \\ Clínica Santa Bárbara Gualaceo \\ Ecuador \\ https://orcid.org/0000-0002-1637-1602 \\ Manuel Alejandro Brito Vizuete \\ drelvisrincones@gmail.com \\ Hospital Moreno Vázquez \\ Ecuador \\ https://orcid.org/0000-0002-8112-8027 \\ Miguel Mateo Sarmiento Álvarez \\ mateo sarmiento@live.com \\ Universidad Católica de Cuenca \\ https://orcid.org/0000-0002-7141-015X \\ Ecuador \\ Johanna Paulina Estrada Cherres \\ johys estrada@hotmail.com \\ Universidad de Cuenca \\ Ecuador \\ https://orcid.org/0000-0002-8112-8027
}

Recibido: 10 de mayo de 2019

Aprobado: 08 de junio de 2019 


\section{RESUMEN}

El síndrome de Wilkie es una enfermedad que se caracteriza por una compresión extrínseca de la tercera porción del duodeno por la arteria mesentérica superior y la aorta. Es de evolución gradual produciendo síntomas obstructivos intestinales altos a largo plazo. El método diagnóstico de elección es la TAC siendo la más efectiva y menos invasiva. El tratamiento de elección al no existir una buena respuesta con el tratamiento conservador, es quirúrgico por medio de una duodenoyeyunostomia aliviando síntomas duodenales y gástricos. El artículo presenta un caso del Síndrome de Arteria Mesentérica Superior que se desarrolla en una mujer de 45 años de edad. La paciente presenta un cuadro clínico de dolor cólico en epigastrio, náuseas y vómitos por varias ocasiones, pérdida de peso de aproximadamente $10 \mathrm{Kg}$, endoscopia digestiva alta, se concluye como pseudo obstrucción intestinal. La Tomografía de abdomen sugiere pinzamiento aortomesenterico del ángulo de Treitz (Síndrome de Wilkie). La paciente no mejora al manejo conservador, por lo que se realiza duodenoyeyuno anastomosis en $Y$ de Roux y se da de alta a los 4 días de la intervención con buena tolerancia oral y a los controles se evidencia ganancia de peso. Transito esofagogastricoduodenal no revela áreas de estenosis o dilatación. Se concluye que el síndrome de Wilkie es una patología infrecuente de baja incidencia, por lo cual es subdiagnosticada, aunque su clínica no es fácil de diferenciar ya que puede confundirse con varias patologías por lo que su diagnóstico se lo realiza tardíamente, siendo la herramienta diagnostica más útil la TAC. Entre las técnicas quirúrgicas de elección, la más apropiada vendría a ser la duodenoyeyunostomia con una mayor tasa de éxito y menor complicaciones.

Descriptores: Síndrome de Wilkie; Duodenoyeyunostomia; Arteria Mesentérica Superior; Síntomas duodenales; Obstrucción Intestinal.

\section{SUMMARY}

Wilkie syndrome is a disease characterized by an extrinsic compression of the third portion of the duodenum by the superior mesenteric artery and the aorta. It is of gradual evolution producing long-term high intestinal obstructive symptoms. The diagnostic method of choice is the CT scan being the most effective and least invasive. The treatment of choice in the absence of a good response with conservative treatment, is surgical through a duodenoyeyunostomy relieving duodenal and gastric symptoms. The article presents a case of Superior Mesenteric Artery Syndrome that develops in a 45year-old woman. The patient presents a clinical picture of colic pain in epigastrium, nausea and vomiting for several occasions, weight loss of approximately $10 \mathrm{Kg}$, upper digestive endoscopy, it is concluded as a pseudo intestinal obstruction. Abdominal tomography suggests aortomesenteric clamping of the Treitz angle (Wilkie syndrome). The patient does not improve conservative management, so duodenoyeyuno 
Revista Arbitrada Interdisciplinaria de Ciencias de la Salud. SALUD Y VIDA

Volumen 3. Número 6. Año 3. Julio - Diciembre 2019

Hecho el depósito de Ley: FA2016000010

ISSN: $2610-8038$

FUNDACIÓN KOINONIA (F.K).

Santa Ana de Coro, Venezuela.

Edisson Andrés Mogollón Guzmán; Manuel Alejandro Brito Vizuete; Miguel Mateo Sarmiento Álvarez; Johanna Paulina Estrada Cherres

anastomosis is performed in $\mathrm{Y}$ of Roux and is discharged 4 days after the intervention with good oral

tolerance and weight gain is evidenced by the controls. Esophagogastricoduodenal transit does not reveal areas of stenosis or dilation. It is concluded that Wilkie syndrome is an uncommon pathology of low incidence, so it is underdiagnosed, although its clinic is not easy to differentiate since it can be confused with several pathologies so that its diagnosis is made late, being the diagnostic tool more useful the TAC. Among the surgical techniques of choice, the most appropriate would be the duodenoyeyunostomy with a higher success rate and fewer complications.

Descriptors: Wilkie syndrome; Duodenoyeyunostomy; Superior Mesenteric Artery; Duodenal symptoms; Intestinal obstruction.

\section{INTRODUCCION}

El síndrome de arteria mesentérica superior o también conocido como síndrome de Wilkie es una patología poco no muy frecuente, originada por la opresión externa de la tercera porción del duodeno por la arteria mesentérica superior (AMS) y la aorta ante la reducción del ángulo aortomesenterico la cual conlleva a producir una obstrucción parcial o completa $(1,2,3,4)$.

El origen de la AMS está ubicado a nivel de L1, orientándose de manera caudal, creando un ángulo de 45 a $60^{\circ}$ en correlación con la aorta. La tercera porción del duodeno atraviesa por el ángulo formado, a nivel de L3-L4, este síndrome se atribuye esencialmente a la perdida de grasa mesentérica que intercede como cojinete entre dichas estructuras $(5,6,7,8,9,10,11,12,13)$.

Puede estar presente en todos los grupos etarios, pero lo más común es que afecte al sexo femenino en una relación 2:1 de 10 a 39 años $(14,15,16,17,18,21)$.

La incidencia exacta de este síndrome no es conocida con exactitud debido a que es poco diagnosticada por su rara frecuencia, además puede existir opresiones duodenales en menor grado que conllevarían a ser un cuadro asintomático. Según revisiones se ha encontrado una incidencia que varía del $0,13 \%$ y que no supera el $1 \%$ $(2,3,6,7,8,9,12,15,16,19,20,21)$, presentando una mortalidad estimada del $33 \%(3,18)$. 
Por lo general las causas que provocan esta patología son adquiridas rara vez congénitas, siendo la perdida abrupta y considerable de peso la principal causa.

Existen múltiples elementos que predisponen el desarrollo de este síndrome: condiciones asociadas a pérdida de peso (SIDA, cáncer, quemaduras extensas, endocrinopatías, malabsorción intestinal, cirugía bariatrica); trastornos de la alimentación (anorexia, bulimia); postoperatorio (cirugía ortopédica, cirugía de columna); traumatismos severos (craneoencefálico, politraumatismos o de la columna vertebral); deformidades como escoliosis $\mathrm{p}$ hiperlordosis pueden desencadenarla $(10,15,16,17,18,19,20,21,22,23)$.

El cuadro clínico común suele ser de inicio progresivo y desarrollo gradual, que puede alargarse por mese o durante años según el grado de compresión, se caracteriza por dolor en epigastrio postprandial inmediato, náuseas, vómitos y sensación de plenitud gástrica. También puede existir una presentación aguda que cursa con síntomas de obstrucción intestinal alta. Estos síntomas pueden provocar que la persona limite su ingesta, disminuyendo de peso, intensificando el cuadro clínico con un a fisiopatología más notoria. El examen físico $n$ es de gran ayuda debido a que es insuficiente para una orientación diagnostica, solo resaltando la notable pérdida de peso y los signos de alteraciones metabólicas producto de vómitos y la deshidratación con la que cursa el paciente. Los síntomas mejoran cuando el paciente se coloca en posición de cubito lateral izquierdo y se agravan en la posición de cubito supino, liberando la presión entre la arteria y el duodeno $(3,5,8,11,14,16,22,23)$.

En el estudio para realizar el diagnostico, los hallazgos de laboratorio solo servirán para definir el estado general del paciente. Desde el uso imagenologico, el examen necesario incluye un tránsito baritado esófago-gastro-duodenal y la angiografía mesentérica, que solo permitirá la apreciación del tracto gastrointestinal y anatomía vascular (1). Los estudios baritados muestran un estómago y duodeno proximal dilatados, un rastro de compresión externa en la tercera porción del duodeno, ondas antiperistálticas en proximidad a la obstrucción, enlentecimiento en el vaciamiento gastroduodenal y sensación de alivio frente al cambio de postural $(3,8,12)$. La 
Revista Arbitrada Interdisciplinaria de Ciencias de la Salud. SALUD Y VIDA

Volumen 3. Número 6. Año 3. Julio - Diciembre 2019

Hecho el depósito de Ley: FA2016000010

ISSN: $2610-8038$

FUNDACIÓN KOINONIA (F.K).

Santa Ana de Coro, Venezuela.

Edisson Andrés Mogollón Guzmán; Manuel Alejandro Brito Vizuete; Miguel Mateo Sarmiento Álvarez; Johanna Paulina Estrada Cherres

angiografía mesentérica muestra el ángulo de la AMS y la disminución de la distancia entre la aorta y la AMS.

En la endoscopia alta se puede evidenciar esofagitis, distención gástrica, ulceras entre otras. $(15,16)$

En la actualidad el estudio imagenologico de preferencia es la TAC, debido a su alto contraste, resolución espacial y temporal, la utilidad de poder realizar reconstrucciones multiplanares $(2,5,17)$.

Una vez diagnosticada la patología, se aconseja inicialmente realizar un tratamiento conservador de 6 semanas por lo menos, estabilizando al paciente, realizando maniobras de descompresión gástrica y región próxima duodenal por medio de colocación de una sonda nasogástrica, se debe además proporcionar una dieta hipercalorica ya sea por vía enteral y/o parenteral, cuando la causa del síndrome esté sujeta con pérdidas bruscas de peso por lo que también se menciona de una hiperalimentacion para poder aumentar la grasa retroperitoneal, el manejo conservador tiene una tasa de éxito del $80 \%$, por lo que al existir recidivas o encontrar fallas se optara por una resolución quirúrgica $(13,15,17,20,21,22)$.

Las opciones quirúrgicas que están disponibles son múltiples, pero la dudenoyeyunostomia (convencional o laparoscópica) latero lateral o en Y de Roux es el procedimiento quirúrgico más aconsejable con una tasa de éxito en el $90 \%$ de los casos siendo la técnica de elección $(8,14,17,20,23)$.

Otros métodos quirúrgicos para el tratamiento son la cirugía de Strong (sección del ligamento de Treitz y amplia movilización del duodeno) y la gastroyeyunostomía, la cual ayuda a la descompresión gástrica pero no a la duodenal desaconsejándose porque ocasiona muchas complicaciones con una tasa de fracaso del $25 \%(1,2,3,6,7,21)$.

\section{CASO CLINICO}

Paciente femenina de 45 años de edad, con antecedente gastritis crónica, quien desde hace 15 días presenta dolor abdominal cólico en epigastrio que se irradia flanco derecho e hipogastrio, acompañado de nausea que lleva al vomito de tipo alimenticio y 
Revista Arbitrada Interdisciplinaria de Ciencias de la Salud. SALUD Y VIDA

Volumen 3. Número 6. Año 3. Julio - Diciembre 2019

Hecho el depósito de Ley: FA2016000010

ISSN: $2610-8038$

FUNDACIÓN KOINONIA (F.K).

Santa Ana de Coro, Venezuela.

Edisson Andrés Mogollón Guzmán; Manuel Alejandro Brito Vizuete; Miguel Mateo Sarmiento Álvarez; Johanna Paulina Estrada Cherres

bilioso por varias ocasiones, al cuadro se sume pérdida de peso de aproximadamente $10 \mathrm{Kg}$, al examen físico dolor a la palpación a nivel de hipogastrio.

Exámenes de laboratorio demuestran proteínas en limites inferiores, ecografía abdominal informa, distensión neumática del marco colonico, en la endoscopia digestiva alta se observa restos alimentarios sólidos en cavidad gástrica, mucosa de fondo, cuerpo, incisura y antro congestiva, con edema importante especialmente en región pre pilórica, en duodeno se progresa hasta la cuarta porción duodenal, observándose pliegues edematosos desde la tercera porción, bulbo y papila duodenal sin lesiones, concluyendo como gastroduodenopatia congestiva edematosa, pseudo obstrucción intestinal,

Tomografía abdominal simple y contrastada informa la presencia de distención y dilatación gástrica, así como del duodeno hasta el ángulo de Treitz, hay retención del contraste oral hasta este nivel, siguieren pinzamiento mesentérico (Síndrome de Wilkie) por pinzamiento aortomesenterico del ángulo de Treitz.

\section{EVOLUCION}

Paciente que no mejora con manejo clínico es intervenida quirúrgicamente 24 horas posteriores a su ingreso, la intervención practicada fue una duodenoyeyunoanastomosis en $\mathrm{Y}$ de Roux, encontrándose duodeno distendido durante su trayecto, anillo fibrotico estenosante a nivel de ángulo de Treitz el cual comprime la cuarta porción del duodeno e impide el paso del tránsito intestinal, yeyuno distal normal A las 48 horas postquirúrgicas se cierra sonda nasogástrica y se inicia con dieta líquida con buena tolerancia retirándose sonda nasogástrica y se extiende a dieta blanda. A los 4 días de la intervención paciente es dada de alta en buenas condiciones y con buena tolerancia oral. A los 14 días de la intervención se evidencia la ganancia de $1 \mathrm{~kg}$ de peso, los exámenes de laboratorio revelan elevación de las proteínas, se realiza transito esofagogastricoduodenal el cual no revela áreas de estenosis o dilatación. 
Revista Arbitrada Interdisciplinaria de Ciencias de la Salud. SALUD Y VIDA

Volumen 3. Número 6. Año 3. Julio - Diciembre 2019

Hecho el depósito de Ley: FA2016000010

ISSN: 2610-8038

FUNDACIÓN KOINONIA (F.K).

Santa Ana de Coro, Venezuela.

Edisson Andrés Mogollón Guzmán; Manuel Alejandro Brito Vizuete; Miguel Mateo Sarmiento Álvarez; Johanna Paulina Estrada Cherres

\section{DISCUSION}

El síndrome de Wilkie constituye una entidad poco frecuente en nuestro medio si bien es más frecuente en mujeres jóvenes, también se puede presentar en niños y mujeres de mediana edad como en nuestro caso.

Generalmente existen factores predisponentes como la pérdida de peso, trastornos de la alimentación, posoperatorio, traumatismo severo, escoliosis, hiperlordosis entre otros. Sin embargo, en algunos casos y como en el nuestro estas condiciones no están presentes. (10)

Dentro del tratamiento lo ideal es tratamiento clínico que incluye

En cuanto al diagnóstico este se torna un verdadero reto ya que sus síntomas son inespecíficos, pero siempre se podrá hacer uso de estudios complementarios quienes nos orientaran el diagnostico así se podría solicitar una endoscopia digestiva alta en donde será evidente retardo en el vaciamiento gastroduodenal condición que cumple en nuestro caso ya que endoscopiasta concluye como una pseudo obstrucción intestinal. La ecografía es útil para medir el ángulo aortomesentérico y la distancia aortomesentérica en niños (3)

\section{CONCLUSION}

El síndrome de Arteria Mesentérica Superior o Síndrome de Wilkie es una patología infrecuente de baja incidencia, por lo cual es subdiagnosticada, aunque su clínica no es fácil de diferenciar ya que puede confundirse con varias patologías por lo que su diagnóstico se lo realiza tardíamente, siendo la herramienta diagnostica más útil la TAC. Entre las técnicas quirúrgicas la de elección vendría a ser la duodenoyeyunostomia con una mayor tasa de éxito y menor complicaciones. 


\section{REFERENCIAS CONSULTADAS}

1. García-Frade-Ruiz LF, Mundo-Gallardo LF, Solís-Ayala E. Síndrome de Wilkie. Med Int Méx [internet]. 2017 mar;33(2):254-258. Disponible en: http://www.scielo.org.mx/pdf/mim/v33n2/0186-4866-mim-33-02-00254.pdf

2. Ochoa-Nava R, Guadarrama-Vallejo A, García-Carrizosa Sócrates, Herrera F. Síndrome de la arteria mesentérica superior una causa inusual de obstrucción intestinal incompleta en un paciente pediátrico. Rev Mex Pediatr [internet]. 2017; 84(2): 61-65. Disponible en: http://www.medigraphic.com/pdfs/pediat/sp$\underline{\text { 2017/sp172d.pdf }}$

3. Vásquez-Arango, Jesús N., Durán-Meléndez, Miguel A., Vásquez-Maya, Camilo, Tratamiento mínimamente invasivo del síndrome de Wilkie. Rev Colomb Cir [internet]. 2018;33: 299-306. Disponible en: http://www.scielo.org.co/pdf/rcci/v33n3/2011-7582-rcci-33-03-00299.pdf DOI: $10.30944 / 20117582.75$

4. Yao S-Y, Mikami R, Mikami S. Minimally invasive surgery for superior mesenteric artery syndrome: A case report. World J Gastroenterol. 2015; 21:12970-5. Disponible en: https://www.ncbi.nlm.nih.gov/pmc/articles/PMC4671049/pdf/WJG21-12970.pdf DOI: 10.3748/wjg.v21.i45.12970

5. Rodriguez A, Vidomlansky S, Ferrarotti C, Larrañaga N, Gallo J, Kozima S. Síndrome de la arteria mesentérica superior Presentación de un caso. Rev Argent Radiol. [internet]. 2014;78(2):96-98. Disponible en: https://www.redalyc.org/pdf/3825/382533982007.pdf

DOI:10.1016/j.rard.2014.06.007

6. Castillo-León J, Pacheco-Barzallo F, Ordoñez M, Pacheco-Granda F. Sindrome de wilkie. Revista de la Sociedad de Cirujanos Generales del Perú [internet]. CIRUJANO 2017; 14 (1): 49-54. Disponible en: http://www.scgp.org/revistacirujano/pdf/2017-REVISTA-CIRUJANO.pdf

7. Hermosillo-Cornejo D, Girón-Gidi A, Vélez-Pérez F, Lemus-Ramírez R, MartínezGarza P. Síndrome de Wilkie reporte de un caso. Cirugía y Cirujanos [internet]. 2017;85(1):54-59. en: https://www.sciencedirect.com/science/article/pii/S0009741115002637?via\%3Dih ub DOI: 10.1016/j.circir.2015.08.009

8. Zaraket V, Deeb L. Wilkie's Syndrome or Superior Mesenteric Artery Syndrome. Case Rep Gastroenterol. [internet]. 2015; 9: 194-9. Disponible en: https://www.ncbi.nlm.nih.gov/pubmed/26120301 DOI: 10.1159/000431307 
9. Martins A, Cunha F, Patricio J, Caravana J. Familial superior mesenteric artery syndrome. BMJ Case Rep [internet]. 2016: 13. Disponible en: https://www.ncbi.nlm.nih.gov/pmc/articles/PMC4854144/pdf/bcr-2016-214784.pdf DOI: $10.1136 / \mathrm{bcr}-2016-214784$

10.Gonzales D, Diaz A., Velez F., Lemus I., Marinez P., Síndrome de Wilkie. Reporte de un caso. Cirugía y Cirujanos. 2015. Disponible en: http://dx.doi.org/10.1016/j.circir.2015.08.009 DOI: 10.1016/j.circir.2015.08.009

11.Lee AM, Bai HX, Zou YJ, Wang ZL, Qiu DX, Tang HY, Yang L. Superior Mesenteric Artery Syndrome in a 20 -year-old, Athletic Female with abdominal Pain. Chin Med J, 2015; 128:3260-1. Disponible en: https://www.ncbi.nlm.nih.gov/pmc/articles/PMC4794869/pdf/CMJ-128-3260.pdf DOI: $10.4103 / 0366-6999.170277$

12. Mathenge N, Osiro S, Rodriguez I, Salib C., Shane R.,Loukas M., Superior Mesenteric Artery Syndrome and Its Associated Gastrointestinal implications, Department of Anatomical Sciences, St. George's University, School of Medicine, Grenada, West Indies, 2013 in Wiley Online Library. Disponible en: https://www.ncbi.nlm.nih.gov/pubmed/23959808 DOI: 10.1002/ca.22249

13. Naseem Z., Premaratne G., Hendahewa R., "Less is more": Non operative management of short term superior mesenteric artery syndrome, Department of General Surgery, Caboolture Hospital, McKean Street, 4510, Caboolture, QLD, Australia, Annals of Medicine and Surgery 4 (2015) 428-430. Disponible en: https://www.sciencedirect.com/science/article/pii/S2049080115001107?via\%3Dih ub DOI: 10.1016/j.amsu.2015.10.011

14.Pesce A, Di Marco F, Stissi R, Li Destri G, Puleo S. Wilkie's syndrome An uncommon cause of small bowel obstruction in a young patient. Ann. Ital. Chir. Published online (EP) 2015; 1-4. Disponible en: https://www.researchgate.net/publication/298212717 Wilkie's syndrome An unc ommon cause of small bowel obstruction in a young patient

15. Poblete $\mathrm{F}$, Álvarez $\mathrm{H}$, Arana A. Síndrome de wilkie caso clínico. Acta Médica Costarricense [internet] 2015; 57: 145-148. Disponible en: http://www.redalyc.org/articulo.oa?id=43439778009

16. Grant N. Síndrome de wilkie compresión duodenal extrínseca poco frecuente. Revista médica de Costa Rica y Centroamérica [internet]. 2014; (611): 403 - 405. disponible en: http://www.medigraphic.com/pdfs/revmedcoscen/rmc 2014/rmc143f.pdf 
17.González A, Muñoz A, Peirano A, Rojas S, Henríquez V. Síndrome de la arteria mesentérica superior presentación de un caso y revisión de la literatura. Cir Pediatr [ilnternet].2016; 29: 180-183. Disponible en: https://www.secipe.org/coldata/upload/revista/2016_29-4_180_183.pdf

18. Sun Z, Rodriguez J, McMichael J, Walsh RM, Chalikonda S, Rosenthal RJ, et al. Minimally invasive duodenojejunostomy for superior mesenteric artery syndrome: a case series and review of the literature. Surg Endosc. 2015; 29:1137-44. Disponible en: https://www.ncbi.nlm.nih.gov/pubmed/25701058 DOI: 10.1007/s00464-014-3775-4

19. Ichikawa T, Yamamuro H, Koizumi J, et al. Duodenal emphysema complicated with superior mesenteric artery Tokai J. Exp Clin Med 2015;40:40-43. Disponible en: https://www.ncbi.nlm.nih.gov/pubmed/26150182

20. Alvarenga A, Espinheira C, Guerra P, Garcia M, Abreu M, Campos M. Superior mesenteric artery síndrome weight loss can be a problema, weight gain can be a solution. Disponible en: https://www.ncbi.nlm.nih.gov/pmc/articles/PMC5553372/ DOI: $10.1159 / 000450898$

21.Pottorf BJ, Husain FA, Hollis HW, Lin E- Laparoscopic Management of Duodenal Obstruction Resulting From Superior Mesenteric Artery Syndrome. JAMA Surg [internet]. 2014;149(12):1319-22. Disponible en: https://www.ncbi.nlm.nih.gov/pubmed/25353279

DOI: 10.1001/jamasurg.2014.1409.

22. Chung A, Smith LI, McMurran A, Ali A. Laparoscopic gastrojejunostomy for superior mesenteric artery syndrome in a patient with Parkinson's disease. Scott Med J. 2016; 61:51-5. Disponible en: https://www.ncbi.nlm.nih.gov/pubmed/26610793

DOI: $10.1177 / 0036933015619290$

23. Alsulaimy M, Tashiro J, Perez EA, Sola JE. Laparoscopic Ladd's procedure for superior mesenteric artery syndrome. J Pediatr Surg. 2014; 49:1533-5. Disponible en: https://www.ncbi.nlm.nih.gov/pubmed/25280662 DOI: 10.1016/j.jpedsurg.2014.07.008 


\section{REFERENCES CONSULTED}

1. García-Frade-Ruiz LF, Mundo-Gallardo LF, Solís-Ayala E. Wilkie syndrome. Med Int Méx [internet]. 2017 Mar; 33 (2): 254-258. Available at: http://www.scielo.org.mx/pdf/mim/v33n2/0186-4866-mim-33-02-00254.pdf

2. Ochoa-Nava R, Guadarrama-Vallejo A, García-Carrizosa Socrates, Herrera F. Upper mesenteric artery syndrome an unusual cause of incomplete intestinal obstruction in a pediatric patient. Rev Mex Pediatr [internet]. 2017; 84 (2): 61-65. Available at: http://www.medigraphic.com/pdfs/pediat/sp-2017/sp172d.pdf

3. Vásquez-Arango, Jesús N., Durán-Meléndez, Miguel A., Vásquez-Maya, Camilo, Minimally invasive treatment of Wilkie syndrome. Rev Colomb Cir [internet]. 2018; 33: 299-306. Available at: http://www.scielo.org.co/pdf/rcci/v33n3/2011-7582-rcci33-03-00299.pdf DOI: 10.30944 / 20117582.75

4. Yao S-Y, Mikami R, Mikami S. Minimally invasive surgery for superior mesenteric artery syndrome: A case report. World J Gastroenterol. 2015; 21: 12970-5. Available at: https://www.ncbi.nlm.nih.gov/pmc/articles/PMC4671049/pdf/WJG21-12970.pdf DOI: 10.3748 / wjg.v21.i45.12970

5. Rodriguez A, Vidomlansky S, Ferrarotti C, Larrañaga N, Gallo J, Kozima S. Upper mesenteric artery syndrome Case report. Rev Argent Radiol. [Internet]. 2014; 78 (2): 96-98. Available at: https://www.redalyc.org/pdf/3825/382533982007.pdf DOI: 10.1016 / j.rard.2014.06.007

6. Castillo-León J, Pacheco-Barzallo F, Ordoñez M, Pacheco-Granda F. Wilkie syndrome. Magazine of the Society of General Surgeons of Peru [internet]. 2017 SURGEON; 14 (1): 49-54. Available at: http://www.scgp.org/revistacirujano/pdf/2017-REVISTA-CIRUJANO.pdf

7. Hermosillo-Cornejo D, Girón-Gidi A, Vélez-Pérez F, Lemus-Ramírez R, MartínezGarza P. Wilkie syndrome case report. Surgery and Surgeons [internet]. 2017; 85 (1): 54-59. Available at: https://www.sciencedirect.com/science/article/pii/S0009741115002637?via\%3Dih ub DOI: 10.1016 / j.circir.2015.08.009

8. Zaraket V, Deeb L. Wilkie's Syndrome or Superior Mesenteric Artery Syndrome. Case Rep Gastroenterol. [Internet]. 2015; 9: 194-9. Available at: https://www.ncbi.nlm.nih.gov/pubmed/26120301 DOI: 10.1159 / 000431307

9. Martins A, Cunha F, Patricio J, Caravan J. Superior family mesenteric artery syndrome. BMJ Case Rep [internet]. 2016: 13. Available at: 
https://www.ncbi.nlm.nih.gov/pmc/articles/PMC4854144/pdf/bcr-2016-214784.pdf DOI: 10.1136 / bcr-2016-214784

10.Gonzales D, Diaz A., Velez F., Lemus I., Marinez P., Wilkie Syndrome. Case report. Surgery and surgeons. 2015. Available at: http://dx.doi.org/10.1016/j.circir.2015.08.009 DOI: 10.1016 / j.circir.2015.08.009

11.Lee AM, Bai HX, Zou YJ, Wang ZL, Quu DX, Tang HY, Yang L. Superior Mesenteric Artery Syndrome in a 20-year-old, Athletic Female with abdominal Pain. Chin Med J, 2015; 128: 3260-1. Available at: https://www.ncbi.nlm.nih.gov/pmc/articles/PMC4794869/pdf/CMJ-128-3260.pdf DOI: 10.4103 / 0366-6999.170277

12. Mathenge N, Osiro S, Rodriguez I, Salib C., Shane R., Loukas M., Superior Mesenteric Artery Syndrome and Its Associated Gastrointestinal implications, Department of Anatomical Sciences, St. George's University, School of Medicine, Grenada, West Indies, 2013 in Wiley Online Library. Available at: https://www.ncbi.nlm.nih.gov/pubmed/23959808 DOI: 10.1002 / ca.22249

13. Naseem Z., Premaratne G., Hendahewa R., "Less is more": Non operative management of short term superior mesenteric artery syndrome, Department of General Surgery, Caboolture Hospital, McKean Street, 4510, Caboolture, QLD, Australia, Annals of Medicine and Surgery 4 (2015) 428-430. Available at: https://www.sciencedirect.com/science/article/pii/S2049080115001107?via\%3Dih ub DOI: 10.1016 / j.amsu.2015.10.011

14.Pesce A, Di Marco F, Stissi R, Li Destri G, Puleo S. Wilkie's syndrome An uncommon cause of small bowel obstruction in a young patient. Ann. Ital. Chir. Published online (EP) 2015; 1-4. Available at: https://www.researchgate.net/publication/298212717 Wilkie's syndrome An unc ommon cause of small bowel obstruction in a young patient

15.Poblete $\mathrm{F}$, Álvarez $\mathrm{H}$, Arana A. Wilkie syndrome clinical case. Costa Rican Medical Act [internet] 2015; 57: 145-148. Available at: http://www.redalyc.org/articulo.oa?id=43439778009

16. Grant N. Wilkie syndrome extrinsic duodenal compression uncommon. Medical magazine of Costa Rica and Central America [internet]. 2014; (611): 403-405. Available at: http://www.medigraphic.com/pdfs/revmedcoscen/rmc 2014 / rmc143f.pdf

17. González A, Muñoz A, Peirano A, Rojas S, Henríquez V. Upper mesenteric artery syndrome case report and literature review. Cir Pediatr [ilnternet]. 2016; 29: 180- 
Revista Arbitrada Interdisciplinaria de Ciencias de la Salud. SALUD Y VIDA

Volumen 3. Número 6. Año 3. Julio - Diciembre 2019

Hecho el depósito de Ley: FA2016000010

ISSN: $2610-8038$

FUNDACIÓN KOINONIA (F.K).

Santa Ana de Coro, Venezuela.

Edisson Andrés Mogollón Guzmán; Manuel Alejandro Brito Vizuete; Miguel Mateo Sarmiento Álvarez; Johanna Paulina Estrada Cherres

183. Available at: https://www.secipe.org/coldata/upload/revista/2016 29$4 \quad 180$ 183.pdf

18.Sun Z, Rodriguez J, McMichael J, Walsh RM, Chalikonda S, Rosenthal RJ, et al. Minimally invasive duodenojejunostomy for superior mesenteric artery syndrome: a case series and review of the literature. Surg Endosc. 2015; 29: 1137-44. Available at: https://www.ncbi.nlm.nih.gov/pubmed/25701058 DOI: 10.1007 / s00464-014-3775-4

19. Ichikawa T, Yamamuro H, Koizumi J, et al. Duodenal emphysema complicated with superior mesenteric artery Tokai J. Exp Clin Med 2015; 40: 40-43. Available at: https://www.ncbi.nlm.nih.gov/pubmed/26150182

20. Alvarenga A, Espinheira C, Guerra P, Garcia M, Abreu M, Campos M. Superior mesenteric artery syndrome weight loss can be a problem, weight gain can be a solution. Available at: https://www.ncbi.nlm.nih.gov/pmc/articles/PMC5553372/ DOI: 10.1159 / 000450898

21.Pottorf BJ, Husain FA, Hollis HW, Lin E. Laparoscopic Management of Duodenal Obstruction Resulting From Superior Mesenteric Artery Syndrome. JAMA Surg [internet]. 2014; 149 (12): 1319-22. Available at: https://www.ncbi.nlm.nih.gov/pubmed/25353279 DOI: 10.1001 / jamasurg.2014.1409.

22.Chung A, Smith LI, McMurran A, Ali A. Laparoscopic gastrojejunostomy for superior mesenteric artery syndrome in a patient with Parkinson's disease. Scott Med J. 2016; 61: 51-5. Available at: https://www.ncbi.nlm.nih.gov/pubmed/26610793 DOI: $10.1177 \quad$ / 0036933015619290

23. Alsulaimy M, Tashiro J, Perez EA, Sola JE. Laparoscopic Ladd's procedure for superior mesenteric artery syndrome. J Pediatr Surg. 2014; 49: 1533-5. Available at: https://www.ncbi.nlm.nih.gov/pubmed/25280662 DOI: 10.1016 / j.jpedsurg.2014.07.008 
Revista Arbitrada Interdisciplinaria de Ciencias de la Salud. SALUD Y VIDA

Volumen 3. Número 6. Año 3. Julio - Diciembre 2019

Hecho el depósito de Ley: FA2016000010

ISSN: 2610-8038

FUNDACIÓN KOINONIA (F.K).

Santa Ana de Coro, Venezuela.

Edisson Andrés Mogollón Guzmán; Manuel Alejandro Brito Vizuete; Miguel Mateo Sarmiento Álvarez; Johanna Paulina Estrada Cherres 